\title{
AN APPROACH TO FREE VIBRATION ANALYSIS OF AXIALLY GRADED BEAMS
}

\author{
Staniseaw Kukla, Jowita Rychlewska \\ Czestochowa University of Technology, Institute of Mathematics, Czestochowa, Poland \\ e-mail: stanislaw.kukla@im.pcz.pl; jowita.rychlewska@im.pcz.pl
}

\begin{abstract}
In this study, the solution to the free vibration problem of axially graded beams with a non-uniform cross-section has been presented. The proposed approach relies on replacing functions characterizing functionally graded beams by piecewise exponential functions. The frequency equation has been derived for axially graded beams divided into an arbitrary number of subintervals. Numerical examples show the influence of the parameters of the functionally graded beams on the free vibration frequencies for different boundary conditions.
\end{abstract}

Keywords: axially graded beam, non-uniform beam, free vibration

\section{Introduction}

Functionally graded materials (FGMs) are a novel class of composites which have continuous variation of material properties from one constituent to another. As a result, they have various advantages over the classical composite laminates. For example, using FGMs, we avoid stress concentrations typical for heterogeneous structures with jump a discontinuity between dissimilar materials. For this reason, FGMs are widely used in mechanical, nuclear, aerospace, biomedical and civil engineering. Simultaneously, because of wide applications of FGMs, it is very important to study static and dynamic analysis of functionally graded structures, such as plates, shells and beams. In this paper, the object of consideration is the problem of free vibration of functionally graded (FG) beams. For FG beams, the gradient variation may be oriented in the axial and/or in the cross-section direction.

The literature on vibration analysis of FG beams with thickness-wise gradient variation is very extensive. For example, Anandrao et al. (2012) made free vibration analysis of functionally graded beams using the principle of virtual work to obtain a finite element system of equations. The variation of material properties across the thickness of the beam was governed by a power law distribution. The same type of variation of the beam properties was also assumed by Sina et al. (2009). They solved the resulting system of ordinary differential equations of free vibration analysis by using an exact method. An analytical solution to study free vibration of exponential functionally graded beams with a single delamination was developed by Liu and Shu (2014). Pradhan and Chakraverty (2013) used the Rayleigh-Ritz model to analyse free vibration of FG beams with material properties that continuously vary in the thickness direction according to the power-law exponent form. This type of gradation was also assumed by Wattanasakulpong and Ungbhakorn (2012). They applied the differential transformation method to solve the governing equation of free vibration of FG beams supported by various types of general boundary conditions. The line spring model to solve the free vibration problem of an exponentially graded cracked beam was employed by Matbuly et al. (2009).

Free vibration analysis for axially graded beams has become more complicated because of the governing equation with variable coefficients. For example, Wu et al. (2005) applied the semi-inverse method to find solutions to the dynamic equation of axially functionally graded 
simply supported beams. Huang and Li (2010) studied free vibration of axially functionally graded beams by using the Fredholm integral equations. Hein and Feklistova (2011) applied the Haar wavelet approach to analyse free vibration of axially functionally graded beams. The differential transform element method and differential quadrature element method of the lowest order were used to solve free vibration and stability problems of FG beams by Shahba and Rajasekaran (2012). The exact solution to free vibration of exponentially axially graded beams was presented by Li et al. (2013). Explicit frequency equations of free vibration of exponentially FG Timoshenko beams were derived by Tang et al. (2014). Huang et al. (2013) presented a new approach to the investigation of free vibration of axially functionally graded Timoshenko beams. By applying auxiliary functions, they transformed the coupled governing equations into a single governing equation. Moreover, there are some studies related with the problem of free vibration of FG beams where the gradation of the material is assumed to be along any of the possible Cartesian coordinates, see Alshorbagy et al. (2011), by Shahba et al. (2013). A review of researches on FG beam type structures can be found in Chauhan and Khan (2014).

In this contribution, we propose a new approach to free vibration analysis of FG beams with arbitrary axial inhomogeneity. The main idea presented in this paper is to approximate an FG beam by an equivalent beam with piece-wise exponentially varying material and geometrical properties. Considerations are carried out in the framework of the Euler-Bernoulli beam theory. Taking into account various boundary conditions associated with clamped, pinned and free ends, numerical solutions are obtained for different functions describing gradient variation of material/geometrical properties of an FG beam. The effectiveness of the proposed approach is confirmed by comparing the obtained numerical results with other numerical solutions available in the existing literature for homogeneous and nonhomogeneous beams. The proposed method is a certain generalization of the approach presented by Kukla and Rychlewska (2014).

\section{Equations of motion}

An axially graded and non-uniform beam of length $\mathrm{L}$ is considered. In this contribution, the material properties and/or cross-section of the beam are assumed to vary continuously along the axial direction. Based on the Euler-Bernoulli beam theory, Lebed and Karnovsky (2000), the governing differential equation is given by

$$
\frac{\partial^{2}}{\partial x^{2}}\left[E(x) I(x) \frac{\partial^{2} w}{\partial x^{2}}\right]+\rho(x) A(x) \frac{\partial^{2} w}{\partial t^{2}}=0 \quad 0<x<L
$$

where $x$ is the axial coordinate, $A(x)$ is the cross-section area, $I(x)$ is the moment of inertia, $E(x)$ denotes the modulus of elasticity, $\rho(x)$ is the material density and $w(x, t)$ is the transverse deflection at the position $x$ and time $t$.

In order to investigate free vibration of the beam, we assume that

$$
w(x, t)=W(x) \sin \omega t
$$

where $W(x)$ is the amplitude of vibration and $\omega$ is the circular frequency of vibration. Substituting (2.2) into (2.1) and introducing the non-dimensional coordinate $\xi=x / L$, we can transform governing equation (2.1) into

$$
\frac{d^{2}}{d \xi^{2}}\left[E(\xi) I(\xi) \frac{d^{2} W}{d \xi^{2}}\right]-L^{4} \omega^{2} \rho(\xi) A(\xi) W=0 \quad 0<\xi<1
$$

In the subsequent analysis, it is assumed that

$$
E(\xi) I(\xi)=d_{0} g(\xi) \quad \rho(\xi) A(\xi)=m_{0} h(\xi) \quad 0<\xi<1
$$


where $d_{0}=E(0) I(0)$ and $m_{0}=\rho(0) A(0)$. Subsequently, we shall approximate the FG beam under consideration by an equivalent beam with piecewise exponentially varying geometrical and material properties, setting

$$
g(\xi) \cong d_{i} \mathrm{e}^{2 \beta_{i} \xi} \quad h(\xi) \cong m_{i} \mathrm{e}^{2 \beta_{i} \xi} \quad \xi_{i-1}<\xi<\xi_{i} \quad i=1, \ldots, n
$$

where $\xi_{0}=0$ and $\xi_{n}=1$. The coefficients $d_{i}, m_{i}, \beta_{i}, i=1, \ldots, n$ we determine by using the following relationships $(i=1, \ldots, n)$

$$
g\left(\xi_{i-1}\right)=d_{i} \mathrm{e}^{2 \beta_{i} \xi_{i-1}} \quad g\left(\xi_{i}\right)=d_{i} \mathrm{e}^{2 \beta_{i} \xi_{i}} \quad g\left(\xi_{0}\right)=1
$$

and $(i=1, \ldots, n)$

$$
h\left(\frac{\xi_{i}+\xi_{i-1}}{2}\right)=m_{i} \mathrm{e}^{\beta_{i}\left(\xi_{i}+\xi_{i-1}\right)} \quad h\left(\xi_{0}\right)=1
$$

Hence $(i=1, \ldots, n)$

$$
\begin{aligned}
\beta_{i} & =\frac{1}{2\left(\xi_{i}-\xi_{i-1}\right)} \ln \frac{g\left(\xi_{i}\right)}{g\left(\xi_{i-1}\right)} \quad d_{i}=g\left(\xi_{i}\right) \mathrm{e}^{-2 \beta_{i} \xi_{i}} \\
m_{i} & =h\left(\frac{\xi_{i}+\xi_{i-1}}{2}\right) \mathrm{e}^{-\beta_{i}\left(\xi_{i}+\xi_{i-1}\right)}
\end{aligned}
$$

We shall also assume that the transverse deflection of the beam has the form

$$
W(\xi)=W_{i}(\xi) \quad \xi_{i-1}<\xi<\xi_{i} \quad i=1, \ldots, n
$$

Hence, the governing system of equations for such a piecewise beam can be expressed by

$$
\frac{d^{2}}{d \xi^{2}}\left[d_{0} d_{i} \mathrm{e}^{2 \beta_{i} \xi} \frac{d^{2} W_{i}}{\partial \xi^{2}}\right]-L^{4} \omega^{2} m_{0} m_{i} \mathrm{e}^{2 \beta_{i} \xi} W_{i}=0 \quad \begin{aligned}
& \xi_{i-1}<\xi<\xi_{i} \\
& i=1, \ldots, n
\end{aligned}
$$

Introducing denotations

$$
\Omega^{2}=\frac{m_{0} m_{1}}{d_{0} d_{1}} L^{4} \omega^{2} \quad \mu_{i}^{2}=\frac{m_{i} d_{1}}{m_{1} d_{i}}
$$

equations (2.10) can be rewritten as

$$
\frac{d^{2}}{d \xi^{2}}\left[\mathrm{e}^{2 \beta_{i} \xi} \frac{d^{2} W_{i}}{d \xi^{2}}\right]-\Omega^{2} \mu_{i}^{2} \mathrm{e}^{2 \beta_{i} \xi} W_{i}=0 \quad \xi_{i-1}<\xi<\xi_{i} \quad i=1, \ldots, n
$$

After some manipulations. equations (2.12) reduce to the form

$$
\frac{d^{4} W_{i}}{d \xi^{4}}+4 \beta_{i} \frac{d^{3} W_{i}}{d \xi^{3}}+4 \beta_{i}^{2} \frac{d^{2} W_{i}}{d \xi^{2}}-\Omega^{2} \mu_{i}^{2} W_{i}=0 \quad \begin{aligned}
& \xi_{i-1}<\xi<\xi_{i} \\
& i=1, \ldots, n
\end{aligned}
$$

The parameters $\beta_{i}$ in equations (2.8) have been determined from the function $g(\cdot)$ corresponding to the stiffness of the beam. These parameters can be determined also by using the function $h(\cdot)$ corresponding to mass of the beam. In this case, we assume that

$$
h\left(\xi_{i-1}\right)=m_{i} \mathrm{e}^{2 \beta_{i} \xi_{i-1}} \quad h\left(\xi_{i}\right)=m_{i} \mathrm{e}^{2 \beta_{i} \xi_{i}} \quad h\left(\xi_{0}\right)=1 \quad i=1, \ldots, n
$$

and

$$
g\left(\frac{\xi_{i}+\xi_{i-1}}{2}\right)=d_{i} \mathrm{e}^{\beta_{i}\left(\xi_{i}+\xi_{i-1}\right)} \quad g\left(\xi_{0}\right)=1 \quad i=1, \ldots, n
$$

Then we have

$$
\begin{aligned}
\beta_{i} & =\frac{1}{2\left(\xi_{i}-\xi_{i-1}\right)} \ln \frac{h\left(\xi_{i}\right)}{h\left(\xi_{i-1}\right)} & m_{i} & =h\left(\xi_{i}\right) \mathrm{e}^{-2 \beta_{i} \xi_{i}} \\
d_{i} & =g\left(\frac{\xi_{i}+\xi_{i-1}}{2}\right) \mathrm{e}^{-\beta_{i}\left(\xi_{i}+\xi_{i-1}\right)} & i & =1, \ldots, n
\end{aligned}
$$

Differential equation (2.13) is valid also for $d_{i}, m_{i}, \beta_{i}$, given by formulae (2.16). 


\section{Solution to the free vibration problem}

On the assumption $\beta_{i}^{2}<\mu_{i} \Omega$, the general solution to equations (2.13) has the form

$$
W_{i}(\xi)=\mathrm{e}^{-\beta_{i} \xi}\left(A_{i} \cos \delta_{i} \xi+B_{i} \sin \delta_{i} \xi+C_{i} \cosh \bar{\delta}_{i} \xi+D_{i} \sinh \bar{\delta}_{i} \xi\right) \quad \xi_{i-1}<\xi<\xi_{i}
$$

where $\delta_{i}=\sqrt{\mu_{i} \Omega-\beta_{i}^{2}}, \bar{\delta}_{i}=\sqrt{\mu_{i} \Omega+\beta_{i}^{2}}, A_{i}, B_{i}, C_{i}, D_{i} \in R, i=1, \ldots, n$.

In order to analyse the free vibration of functionally graded beams, solution (3.1) has to be applied to certain boundary conditions. In this paper we shall consider the following types of boundary conditions:

- clamped-clamped beam (C-C)

$$
W_{1}(0)=0 \quad \frac{d W_{1}}{d \xi}(0)=0 \quad W_{n}(1)=0 \quad \frac{d W_{n}}{d \xi}(1)=0
$$

— pinned-pinned beam (P-P)

$$
W_{1}(0)=0 \quad \frac{d^{2} W_{1}}{d \xi^{2}}(0)=0 \quad W_{n}(1)=0 \quad \frac{d^{2} W_{n}}{d \xi^{2}}(1)=0
$$

— clamped-pinned beam (C-P)

$$
W_{1}(0)=0 \quad \frac{d W_{1}}{d \xi}(0)=0 \quad W_{n}(1)=0 \quad \frac{d^{2} W_{n}}{d \xi^{2}}(1)=0
$$

— pinned-clamped beam (P-C)

$$
W_{1}(0)=0 \quad \frac{d^{2} W_{1}}{d \xi^{2}}(0)=0 \quad W_{n}(1)=0 \quad \frac{d W_{n}}{d \xi}(1)=0
$$

- clamped-free beam (C-F)

$$
W_{1}(0)=0 \quad \frac{d W_{1}}{d \xi}(0)=0 \quad \frac{d^{2} W_{n}}{d \xi^{2}}(1)=0 \quad \frac{d}{d \xi}\left(\mathrm{e}^{2 \beta \xi} \frac{d^{2} W_{n}}{d \xi^{2}}\right)(1)=0
$$

— free-clamped beam (F-C)

$$
\frac{d^{2} W_{1}}{d \xi^{2}}(0)=0 \quad \frac{d}{d \xi}\left(e^{2 \beta \xi} \frac{d^{2} W_{1}}{d \xi^{2}}\right)(0)=0 \quad W_{n}(1)=0 \quad \frac{d W_{n}}{d \xi}(1)=0
$$

The matching conditions between two connecting elements of the piecewise beams satisfy the following continuity conditions

$$
\begin{aligned}
& W_{i}\left(\xi_{i}\right)=W_{i+1}\left(\xi_{i}\right) \\
& \frac{d W_{i}}{d \xi}\left(\xi_{i}\right)=\frac{d W_{i+1}}{d \xi}\left(\xi_{i}\right) \\
& \frac{d^{2} W_{i}}{d \xi^{2}}\left(\xi_{i}\right)=\frac{d^{2} W_{i+1}}{d \xi^{2}}\left(\xi_{i}\right) \\
& \frac{d^{3} W_{i}}{d \xi^{3}}\left(\xi_{i}\right)=\frac{d^{3} W_{i+1}}{d \xi^{3}}\left(\xi_{i}\right) \\
& i=1, \ldots, n-1
\end{aligned}
$$

Substituting functions (3.1) into one of the set of boundary conditions (3.2)-(3.7) and continuity conditions given by equations (3.8), we obtain a system of $4 \mathrm{n}$ equations which can be written in the matrix form

$$
\mathbf{A}(\omega) \mathbf{X}=\mathbf{0}
$$


where $\mathbf{X}=\left[A_{1}, B_{1}, C_{1}, D_{1}, \ldots, A_{n}, B_{n}, C_{n}, D_{n}\right]^{\mathrm{T}}$ and $\mathbf{A}(\omega)=\left[a_{k j}\right]_{4 n \times 4 n}$. The matrix $\mathbf{A}$ can be expressed as

$$
\mathbf{A}(\omega)=\left[\begin{array}{c}
\mathbf{B}_{1} \\
\mathbf{C}_{1} \\
\mathbf{C}_{2} \\
\vdots \\
\mathbf{C}_{n-1} \\
\mathbf{B}_{n}
\end{array}\right]_{4 n \times 4 n}
$$

where the matrices $\mathbf{B}_{1}, \mathbf{B}_{n}$ of size $(2 \times 4 n)$ represent the boundary conditions and matrices $\mathbf{C}_{i}$, $i=1, \ldots, n-1$ of size $(4 \times 4 n)$ represent the continuity conditions. The matrices associated with the boundary conditions corresponding to the four kinds of end supports can be written as follows:

- clamped-clamped beams

$$
\begin{aligned}
\mathbf{B}_{1} & =\left[\begin{array}{ccccccc}
1 & 0 & 1 & 0 & 0 & \cdots & 0 \\
-\beta_{1} & \delta_{1} & -\beta_{1} & \bar{\delta}_{1} & 0 & \cdots & 0
\end{array}\right]_{2 \times 4 n} \\
\mathbf{B}_{n} & =\left[\begin{array}{llllllll}
0 & \cdots & 0 & \cos \delta_{n} & \sin \delta_{n} & \cosh \bar{\delta}_{n} & \sinh \bar{\delta}_{n} \\
0 & \cdots & 0 & a_{4 n, 4 n-3} & a_{4 n, 4 n-2} & a_{4 n, 4 n-1} & a_{4 n, 4 n}
\end{array}\right]_{2 \times 4 n}
\end{aligned}
$$

where

$$
\begin{aligned}
& a_{4 n, 4 n-3}=-\beta_{n} \cos \delta_{n}-\delta_{n} \sin \delta_{n} \\
& a_{4 n, 4 n-2}=-\beta_{n} \sin \delta_{n}+\delta_{n} \cos \delta_{n} \\
& a_{4 n, 4 n-1}=-\beta_{n} \cosh \bar{\delta}_{n}+\bar{\delta}_{n} \sinh \bar{\delta}_{n} \\
& a_{4 n, 4 n}=-\beta_{n} \sinh \bar{\delta}_{n}+\bar{\delta}_{n} \cosh \bar{\delta}_{n}
\end{aligned}
$$

— pinned-pinned beams

$$
\begin{aligned}
\mathbf{B}_{1} & =\left[\begin{array}{ccccccc}
1 & 0 & 1 & 0 & 0 & \cdots & 0 \\
\beta_{1}^{2}-\delta_{1}^{2} & -2 \beta_{1} \delta_{1} & \beta_{1}^{2}+\bar{\delta}_{1}^{2} & -2 \beta_{1} \bar{\delta}_{1} & 0 & \cdots & 0
\end{array}\right]_{2 \times 4 n} \\
\mathbf{B}_{n} & =\left[\begin{array}{cccccccc}
0 & \cdots & 0 & \cos \delta_{n} & \sin \delta_{n} & \cosh \bar{\delta}_{n} & \sinh \bar{\delta}_{n} \\
0 & \cdots & 0 & a_{4 n, 4 n-3} & a_{4 n, 4 n-2} & a_{4 n, 4 n-1} & a_{4 n, 4 n}
\end{array}\right]_{2 \times 4 n}
\end{aligned}
$$

where

$$
\begin{aligned}
& a_{4 n, 4 n-3}=2 \beta_{n} \delta_{n} \sin \delta_{n}+\left(\beta_{n}^{2}-\delta_{n}^{2}\right) \cos \delta_{n} \\
& a_{4 n, 4 n-2}=-2 \beta_{n} \delta_{n} \cos \delta_{n}+\left(\beta_{n}^{2}-\delta_{n}^{2}\right) \sin \delta_{n} \\
& a_{4 n, 4 n-1}=-2 \beta_{n} \bar{\delta}_{n} \sinh \bar{\delta}_{n}+\left(\beta_{n}^{2}+\bar{\delta}_{n}^{2}\right) \cosh \bar{\delta}_{n} \\
& a_{4 n, 4 n}=-2 \beta_{n} \bar{\delta}_{n} \cosh \bar{\delta}_{n}+\left(\beta_{n}^{2}+\bar{\delta}_{n}^{2}\right) \sinh \bar{\delta}_{n}
\end{aligned}
$$

— free-clamped beams

$$
\mathbf{B}_{1}=\left[\begin{array}{ccccccc}
\beta_{1}^{2}-\delta_{1}^{2} & -2 \beta_{1} \delta_{1} & \beta_{1}^{2}+\bar{\delta}_{1}^{2} & -2 \beta_{1} \bar{\delta}_{1} & 0 & \cdots & 0 \\
\beta_{1}^{3}+\beta_{1} \delta_{1}^{2} & -\beta_{1}^{2} \delta_{1}-\delta_{1}^{3} & \beta_{1}^{3}-\beta_{1} \bar{\delta}_{1}^{2} & -\beta_{1}^{2} \bar{\delta}_{1}+\bar{\delta}_{1}^{3} & 0 & \cdots & 0
\end{array}\right]_{2 \times 4 n}
$$

the matrix $\mathbf{B}_{n}$ is given by $(3.11)_{2}$ 
- clamped-free beams

the matrix $\mathbf{B}_{1}$ is given by $(3.11)_{1}$

$$
\mathbf{B}_{n}=\left[\begin{array}{ccccccc}
0 & \cdots & 0 & a_{4 n-1,4 n-3} & a_{4 n-1,4 n-2} & a_{4 n-1,4 n-1} & a_{4 n-1,4 n} \\
0 & \cdots & 0 & a_{4 n, 4 n-3} & a_{4 n, 4 n-2} & a_{4 n, 4 n-1} & a_{4 n, 4 n}
\end{array}\right]_{2 \times 4 n}
$$

where

$$
\begin{aligned}
& a_{4 n-1,4 n-3}=2 \beta_{n} \delta_{n} \sin \delta_{n}+\left(\beta_{n}^{2}-\delta_{n}^{2}\right) \cos \delta_{n} \\
& a_{4 n-1,4 n-2}=-2 \beta_{n} \delta_{n} \cos \delta_{n}+\left(\beta_{n}^{2}-\delta_{n}^{2}\right) \sin \delta_{n} \\
& a_{4 n-1,4 n-1}=-2 \beta_{n} \bar{\delta}_{n} \sinh \bar{\delta}_{n}+\left(\beta_{n}^{2}+\bar{\delta}_{n}^{2}\right) \cosh \bar{\delta}_{n} \\
& a_{4 n-1,4 n}=-2 \beta_{n} \bar{\delta}_{n} \cosh \bar{\delta}_{n}+\left(\beta_{n}^{2}+\bar{\delta}_{n}^{2}\right) \sinh \bar{\delta}_{n} \\
& a_{4 n, 4 n-3}=\left(\beta_{n}^{2} \delta_{n}+\delta_{n}^{3}\right) \sin \delta_{n}+\left(\beta_{n}^{3}+\beta_{n} \delta_{n}^{2}\right) \cos \delta_{n} \\
& a_{4 n, 4 n-2}=-\left(\beta_{n}^{2} \delta_{n}+\delta_{n}^{3}\right) \cos \delta_{n}+\left(\beta_{n}^{3}+\beta_{n} \delta_{n}^{2}\right) \sin \delta_{n} \\
& a_{4 n, 4 n-1}=\left(-\beta_{n}^{2} \bar{\delta}_{n}+\bar{\delta}_{n}^{3}\right) \sinh \bar{\delta}_{n}+\left(\beta_{n}^{3}-\beta_{n} \bar{\delta}_{n}^{2}\right) \cosh \bar{\delta}_{n} \\
& a_{4 n, 4 n}=\left(-\beta_{n}^{2} \bar{\delta}_{n}+\bar{\delta}_{n}^{3}\right) \cosh \bar{\delta}_{n}+\left(\beta_{n}^{3}-\beta_{n} \bar{\delta}_{n}^{2}\right) \sinh \bar{\delta}_{n}
\end{aligned}
$$

For clamped-pinned and pinned-clamped beams, the matrices $\mathbf{B}_{1}, \mathbf{B}_{n}$ are given by equations $(3.11)_{1}-(3.13)_{2}$ and $(3.13)_{1}-(3.11)_{2}$, respectively. The matrices associated with the continuity conditions are represented by

$$
\mathbf{C}_{i}=\left[\begin{array}{ccccccccc}
0 & \cdots & 0 & a_{4 i-1,4 i-3} & \cdots & a_{4 i-1,4 i+4} & 0 & \cdots & 0 \\
0 & \cdots & 0 & a_{4 i, 4 i-3} & \cdots & a_{4 i, 4 i+4} & 0 & \cdots & 0 \\
0 & \cdots & 0 & a_{4 i+1,4 i-3} & \cdots & a_{4 i+1,4 i+4} & 0 & \cdots & 0 \\
0 & \cdots & 0 & a_{4 i+2,4 i-3} & \cdots & a_{4 i+2,4 i+4} & 0 & \cdots & 0
\end{array}\right]_{4 \times 4 n} i=1, \ldots, n-1
$$

The non-zero elements of these matrices are given in Appendix.

The determinant of the matrix $\mathbf{A}$ has to vanish for a non-trivial solution of equation (3.9) to exist. The frequency equation

$$
\operatorname{det} \mathbf{A}(\omega)=0
$$

is then solved numerically using an approximate method.

\section{Numerical results}

The numerical computations have been carried out for an FG beam which was divided into $n$ segments of the same length. The functions $g(\cdot), h(\cdot)$ introduced into equations (2.4) are assumed in the form $g(\xi)=(1+\gamma \xi)^{\alpha}, h(\xi)=1+\gamma \xi$. In the computations, the formulae given by equation (2.8) have been used. The first three non-dimensional free vibration frequencies obtained in the present study for $n=100$ are listed in Tables 1 and 2 in comparison with those presented by Huang and Li (2010) and calculated by using a power series expansion. From Tables 1-2, it can be seen that the present results are in good agreement with the existing results. For $\gamma=0$, we have the case of a homogeneous beam. It is seen in Tables 1 and 2 that in this case the agreement is excellent. 
Table 1. The first three non-dimensional free vibration frequencies for $g(\xi)=(1+\gamma \xi)^{3}$, $h(\xi)=1+\gamma \xi$, clamped-clamped beam

\begin{tabular}{|c|c|c|c|}
\hline$\gamma$ & Power series method & Huang and Li (2010) & Present study \\
\hline \hline \multirow{3}{*}{0.1} & 21.2409777868 & 21.24097778688 & 21.242905 \\
\cline { 2 - 4 } & 58.5500545739 & 58.55005461550 & 58.567526 \\
\cline { 2 - 4 } & 114.780241659 & 114.78027750905 & 114.824704 \\
\hline 0 & 22.3732854478 & 22.37328544806 & 22.373285 \\
\cline { 2 - 4 } & 61.6728228676 & 61.67282294761 & 61.672823 \\
\cline { 2 - 4 } & 120.903391727 & 120.90340027002 & 120.903392 \\
\hline 0.1 & 23.4796072481 & 23.47960724845 & 23.460013 \\
\cline { 2 - 4 } & 64.7210676329 & 64.72106768601 & 64.678046 \\
\cline { 2 - 4 } & 126.878016311 & 126.87805071630 & 126.802905 \\
\hline 0.2 & 24.5634175322 & 24.5634175326 & 24.508817 \\
\cline { 2 - 4 } & 67.7047553171 & 67.7047553184 & 67.596273 \\
\cline { 2 - 4 } & 132.723976757 & 132.7240684027 & 132.546612 \\
\hline
\end{tabular}

Table 2. The first three non-dimensional free vibration frequencies for $g(\xi)=(1+\gamma \xi)^{3}$, $h(\xi)=1+\gamma \xi$, clamped-pinned beam

\begin{tabular}{|c|c|c|c|}
\hline$\gamma$ & Power series method & Huang and Li (2010) & Present study \\
\hline \hline-0.1 & 14.8488960557 & 14.84889605539 & 14.844562 \\
\cline { 2 - 4 } & 47.6370371901 & 47.63703719174 & 47.647237 \\
\cline { 2 - 4 } & 99.171635183 & 99.17165323722 & 99.206918 \\
\hline 0 & 15.4182057169 & 15.41820571698 & 15.418206 \\
\cline { 2 - 4 } & 49.964862032 & 49.96486203816 & 49.964862 \\
\cline { 2 - 4 } & 104.247696458 & 104.24770194514 & 104.247696 \\
\hline 0.1 & 15.968709884 & 15.96870988416 & 15.950015 \\
\cline { 2 - 4 } & 52.2372268871 & 52.23722689317 & 52.198883 \\
\cline { 2 - 4 } & 109.202352455 & 109.20235370558 & 109.134912 \\
\hline 0.2 & 16.5028988943 & 16.50289889399 & 16.445277 \\
\cline { 2 - 4 } & 54.4614625302 & 54.46146253076 & 54.360368 \\
\cline { 2 - 4 } & 114.051623344 & 114.05163085534 & 113.888586 \\
\hline
\end{tabular}

The effects of parameters $\alpha, \gamma$ and the number of segments $n$ on the first three nondimensional frequencies for different boundary conditions are presented in Tables 3-5. It can be observed that an increase in the value of the parameter $\alpha$ causes an increase in the difference between the results obtained for $n=5, n=10$ and $n=20$, respectively.

Figure 1 presents the first free vibration frequencies calculated for the functions $g(\xi)=(1+\gamma \xi)^{\alpha}$ and $h(\xi)=1+\gamma \xi$ for $\alpha=1, \alpha=2$ and $\alpha=3$. The calculations have been performed for six types of boundary conditions. It can be noticed that variation of the parameter $\gamma$ has a significant effect on the free vibration frequency. For the clamped-free beams, the greatest impact of the parameter $\gamma$ occurs for $\alpha=1$, and for all the other boundary conditions under considerations it is for $\alpha=3$. For the clamped-clamped and pinned-pinned beams, the differences between the values of free vibration frequencies for $\alpha=1$ are negligible. 
Table 3. The first non-dimensional free vibration frequency for different boundary conditions, $g(\xi)=(1+\gamma \xi)^{\alpha}, h(\xi)=1+\gamma \xi$

\begin{tabular}{|c|c|c|c|c|c|c|c|}
\hline \multirow{2}{*}{ BC } & \multirow{2}{*}{$n$} & \multicolumn{2}{|c|}{$\alpha=1$} & \multicolumn{2}{|c|}{$\alpha=2$} & \multicolumn{2}{|c|}{$\alpha=3$} \\
\cline { 3 - 8 } & & $\gamma=-0.5$ & $\gamma=0.5$ & $\gamma=-0.5$ & $\gamma=0.5$ & $\gamma=-0.5$ & $\gamma=0.5$ \\
\hline \hline \multirow{3}{*}{ C-C } & 5 & 22.03982 & 22.264535 & 19.265636 & 24.165700 & 16.814251 & 26.214186 \\
\cline { 2 - 8 } & 10 & 22.051059 & 22.262618 & 19.048822 & 24.459508 & 16.441212 & 26.863566 \\
\cline { 2 - 8 } & 20 & 22.053796 & 22.261942 & 18.935593 & 24.609820 & 16.247100 & 27.197036 \\
\hline P-P & 5 & 9.583045 & 9.773619 & 8.177437 & 10.524969 & 6.896407 & 11.294071 \\
\cline { 2 - 8 } & 10 & 9.588353 & 9.772740 & 8.086959 & 10.653015 & 6.746380 & 11.574244 \\
\cline { 2 - 8 } & 20 & 9.589777 & 9.772464 & 8.039484 & 10.718554 & 6.667729 & 11.718081 \\
\hline C-P & 5 & 15.838604 & 14.907051 & 14.080008 & 15.909067 & 12.483974 & 16.939622 \\
\cline { 2 - 8 } & 10 & 15.845921 & 14.905829 & 13.922110 & 16.102144 & 12.209467 & 17.358429 \\
\cline { 2 - 8 } & 20 & 15.847897 & 14.905405 & 13.839671 & 16.201108 & 12.066042 & 17.573873 \\
\hline P-C & 5 & 14.448440 & 15.749301 & 12.216596 & 17.289925 & 10.244436 & 18.957895 \\
\cline { 2 - 8 } & 10 & 14.456396 & 15.747708 & 12.079634 & 17.500534 & 10.017769 & 19.429126 \\
\cline { 2 - 8 } & 20 & 14.458368 & 15.747212 & 12.008080 & 17.608138 & 9.899795 & 19.670529 \\
\hline C-F & 5 & 4.379586 & 3.121248 & 4.321434 & 3.188805 & 4.234717 & 3.251220 \\
\cline { 2 - 8 } & 10 & 4.380502 & 3.120991 & 4.252879 & 3.232930 & 4.103112 & 3.342365 \\
\cline { 2 - 8 } & 20 & 4.380745 & 3.120903 & 4.222757 & 3.254157 & 4.045646 & 3.386566 \\
\hline \multirow{2}{*}{ F-C } & 5 & 2.887779 & 3.995266 & 2.290903 & 4.643804 & 1.807925 & 5.384977 \\
\cline { 2 - 8 } & 10 & 2.889157 & 3.994728 & 2.270108 & 4.689088 & 1.774671 & 5.492166 \\
\cline { 2 - 8 } & 20 & 2.889508 & 3.994563 & 2.257891 & 4.715110 & 1.755475 & 5.553767 \\
\hline
\end{tabular}

Table 4. The second non-dimensional free vibration frequency for different boundary conditions, $g(\xi)=(1+\gamma \xi)^{\alpha}, h(\xi)=1+\gamma \xi$

\begin{tabular}{|c|c|c|c|c|c|c|c|}
\hline \multirow{2}{*}{ BC } & \multirow{2}{*}{$n$} & \multicolumn{2}{|c|}{$\alpha=1$} & \multicolumn{2}{|c|}{$\alpha=2$} & \multicolumn{2}{|c|}{$\alpha=3$} \\
\cline { 3 - 8 } & & $\gamma=-0.5$ & $\gamma=0.5$ & $\gamma=-0.5$ & $\gamma=0.5$ & $\gamma=-0.5$ & $\gamma=0.5$ \\
\hline \hline C-C & 5 & 61.187699 & 61.527290 & 53.563835 & 66.810777 & 46.705060 & 72.453327 \\
\cline { 2 - 8 } & 10 & 61.220229 & 61.522622 & 52.964685 & 67.624175 & 45.679608 & 74.250130 \\
\cline { 2 - 8 } & 20 & 61.227661 & 61.520754 & 52.649784 & 68.039773 & 45.140471 & 75.171899 \\
\hline P-P & 5 & 39.248490 & 39.415763 & 34.281704 & 42.767233 & 29.772507 & 46.312345 \\
\cline { 2 - 8 } & 10 & 39.265508 & 39.411680 & 33.896833 & 43.288075 & 29.121704 & 47.465112 \\
\cline { 2 - 8 } & 20 & 39.270192 & 39.410436 & 33.695664 & 43.554164 & 28.779150 & 48.054717 \\
\hline C-P & 5 & 50.300388 & 49.461958 & 44.282315 & 53.482420 & 38.806190 & 57.735593 \\
\cline { 2 - 8 } & 10 & 50.321872 & 49.457835 & 43.792788 & 54.127769 & 37.971984 & 59.156518 \\
\cline { 2 - 8 } & 20 & 50.327702 & 49.456336 & 43.532945 & 54.460171 & 37.525512 & 59.890410 \\
\hline P-C & 5 & 48.939014 & 50.267141 & 42.517999 & 54.777959 & 36.759687 & 59.599505 \\
\cline { 2 - 8 } & 10 & 48.964462 & 50.262091 & 42.035739 & 55.451783 & 35.941623 & 61.097469 \\
\cline { 2 - 8 } & 20 & 48.970497 & 50.260490 & 41.785685 & 55.792915 & 35.517228 & 61.857034 \\
\hline C-F & 5 & 23.381744 & 21.158807 & 21.233450 & 22.452525 & 19.191540 & 23.771357 \\
\cline { 2 - 8 } & 10 & 23.393474 & 21.157385 & 20.890872 & 22.761849 & 18.565383 & 24.434588 \\
\cline { 2 - 8 } & 20 & 23.396111 & 21.156800 & 20.740129 & 22.911071 & 18.295994 & 24.757241 \\
\hline \multirow{2}{*}{ F-C } & 5 & 20.476134 & 22.847483 & 17.187639 & 25.342189 & 14.317750 & 28.060961 \\
\cline { 2 - 8 } & 10 & 20.488032 & 22.845673 & 17.036003 & 25.587931 & 14.064074 & 28.609593 \\
\cline { 2 - 8 } & 20 & 20.490837 & 22.844949 & 16.945280 & 25.728924 & 13.914191 & 28.926634 \\
\hline
\end{tabular}


Table 5. The third non-dimensional free vibration frequency for different boundary conditions, $g(\xi)=(1+\gamma \xi)^{\alpha}, h(\xi)=1+\gamma \xi$

\begin{tabular}{|c|c|c|c|c|c|c|c|}
\hline \multirow{2}{*}{ BC } & \multirow{2}{*}{$n$} & \multicolumn{2}{|c|}{$\alpha=1$} & \multicolumn{2}{|c|}{$\alpha=2$} & \multicolumn{2}{|c|}{$\alpha=3$} \\
\cline { 2 - 8 } & & $\gamma=-0.5$ & $\gamma=0.5$ & $\gamma=-0.5$ & $\gamma=0.5$ & $\gamma=-0.5$ & $\gamma=0.5$ \\
\hline \hline \multirow{6}{*}{ C-C } & 5 & 120.319511 & 120.746274 & 105.344423 & 131.127496 & 91.681928 & 142.145173 \\
\cline { 2 - 8 } & 10 & 120.392769 & 120.740300 & 104.218260 & 132.739432 & 89.853340 & 145.728137 \\
\cline { 2 - 8 } & 20 & 120.407372 & 120.736681 & 103.598604 & 133.555211 & 88.792805 & 147.537078 \\
\hline P-P & 5 & 88.594258 & 88.779880 & 77.531290 & 96.389774 & 67.426814 & 104.429449 \\
\cline { 2 - 8 } & 10 & 88.629715 & 88.770369 & 76.684167 & 97.575681 & 66.023866 & 107.072172 \\
\cline { 2 - 8 } & 20 & 88.639792 & 88.767515 & 76.229088 & 98.175746 & 65.248530 & 108.403315 \\
\hline C-P & 5 & 104.554645 & 103.740552 & 91.766692 & 112.472339 & 80.084560 & 121.703952 \\
\cline { 2 - 8 } & 10 & 104.597719 & 103.733256 & 90.815730 & 113.815304 & 78.503258 & 124.685866 \\
\cline { 2 - 8 } & 20 & 104.609566 & 103.730098 & 90.278366 & 114.513546 & 77.584066 & 126.231330 \\
\hline P-C & 5 & 103.144606 & 104.556740 & 90.048791 & 113.706807 & 78.140870 & 123.417530 \\
\cline { 2 - 8 } & 10 & 103.202283 & 104.546600 & 89.029672 & 115.147269 & 76.462403 & 126.629660 \\
\cline { 2 - 8 } & 20 & 103.214761 & 104.543267 & 88.499082 & 115.856814 & 75.558342 & 128.206906 \\
\hline C-F & 5 & 62.982515 & 60.852831 & 56.140476 & 65.522940 & 49.819399 & 70.432539 \\
\cline { 2 - 8 } & 10 & 63.020704 & 60.849312 & 55.256707 & 66.417417 & 48.227924 & 72.379515 \\
\cline { 2 - 8 } & 20 & 63.028371 & 60.847560 & 54.856960 & 66.851872 & 47.524147 & 73.333012 \\
\hline F-C & 5 & 60.136355 & 62.488331 & 51.918494 & 68.523131 & 44.593816 & 75.023941 \\
\cline { 2 - 8 } & 10 & 60.171203 & 62.485112 & 51.449765 & 69.200653 & 43.789492 & 76.516820 \\
\cline { 2 - 8 } & 20 & 60.178811 & 62.483270 & 51.173617 & 69.581994 & 43.319244 & 77.363991 \\
\hline
\end{tabular}

The presented numerical results have been obtained by using coefficients $d_{i}, m_{i}, \beta_{i}$, $i=1, \ldots, n$, given by equations (2.8). Numerical computations show that the application of equations (2.16) leads to results which are in good agreement with the obtained by using equations (2.8).

\section{Conclusions}

In the paper, a solution to the free vibration problem of axially functionally graded beams is presented. An exact solution is derived for axially piece-wise exponential graded beams. The frequency equation for beams with various combinations of clamped, pinned and free ends has been obtained. In this approach, the distributed parameters which describe continuous axial changes of the material properties of the beam are approximated by piecewise exponential functions. The non-dimensional free vibration frequencies for a chosen function characterizing the functionally graded beams have been numerically computed. An improvement of the accuracy of the numerical results for a larger number of beam subsections applied in the method has been demonstrated. A high agreement of the numerical results obtained by using the presented method with the results obtained by using the power series method as well as with results given by other authors has also been observed. The numerical investigation shows that the beam stiffness distribution in the axial direction significantly effects free vibration frequencies of the system. 

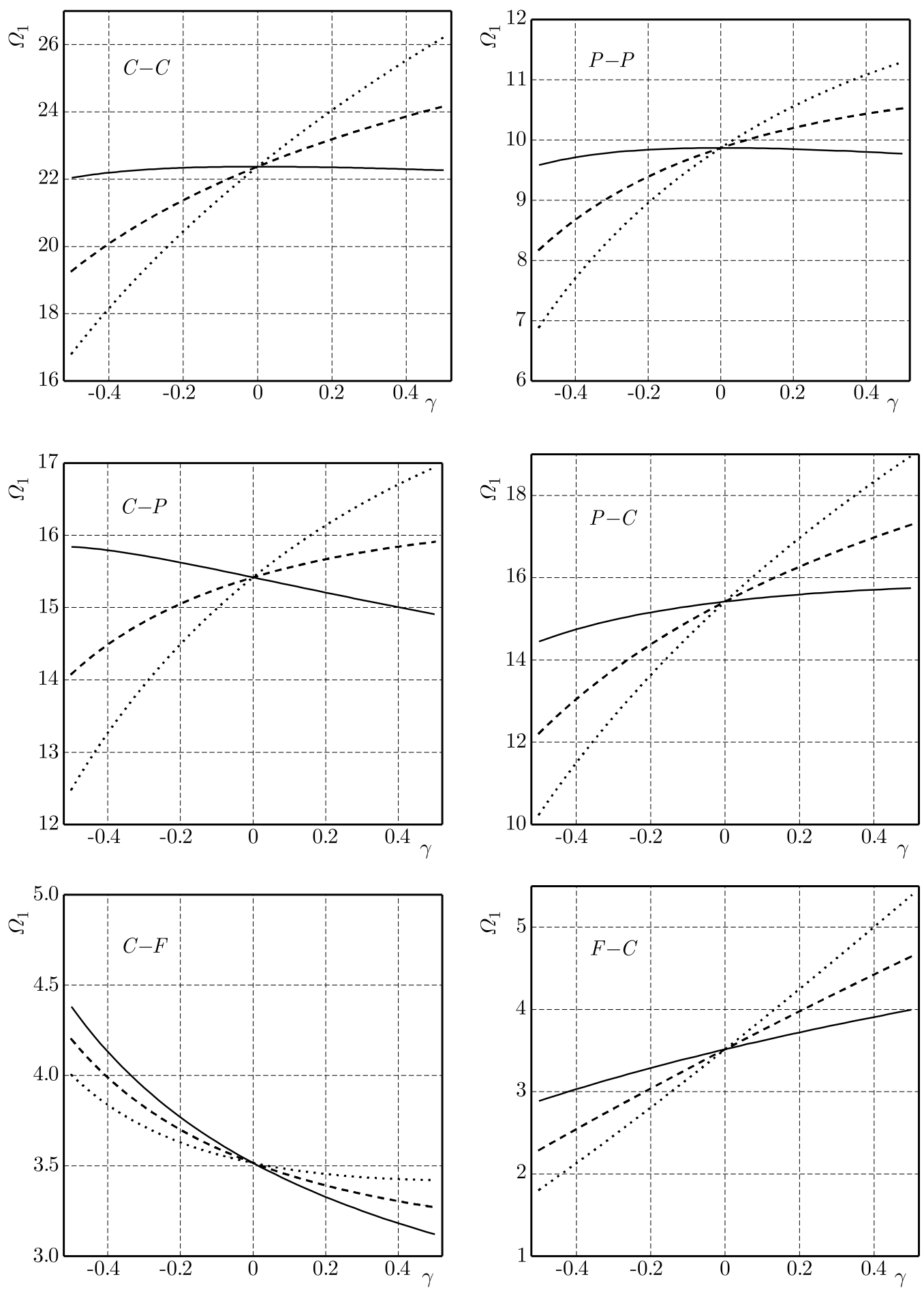

Fig. 1. The first non-dimensional free vibration frequency as a function of $\gamma$ for $\alpha=1$ (solid line), $\alpha=2$ (dashed line), $\alpha=3$ (dotted line) for different boundary conditions

\section{Appendix}

Let us denote $e x i=\mathrm{e}^{\xi_{i}}\left(\beta_{i+1}-\beta_{i}\right), c i=\cos \left(\delta_{i} \xi_{i}\right), s i=\sin \left(\delta_{i} \xi_{i}\right), c h i=\cosh \left(\bar{\delta}_{i} \xi_{i}\right), s h i=\sinh \left(\bar{\delta}_{i} \xi_{i}\right)$, $c i 1=\cos \left(\delta_{i+1} \xi_{i}\right), \operatorname{si1}=\sin \left(\delta_{i+1} \xi_{i}\right), \operatorname{ch} i 1=\cosh \left(\bar{\delta}_{i+1} \xi_{i}\right), \operatorname{sh} i 1=\sinh \left(\bar{\delta}_{i+1} \xi_{i}\right)$.

The non-zero elements of the matrix $\mathbf{C}_{i}, i=1, \ldots, n-1$, which occur in equation (3.18) are given by 


$$
\begin{aligned}
& a_{4 i-1,4 i-3}=e x i \cdot c i \quad a_{4 i-1,4 i-2}=e x i \cdot s i \quad a_{4 i-1,4 i-1}=e x i \cdot c h i \\
& a_{4 i-1,4 i}=e x i \cdot s h i \quad a_{4 i-1,4 i+1}=-c i 1 \quad a_{4 i-1,4 i+2}=-s i 1 \\
& a_{4 i-1,4 i+3}=- \text { chi1 } \quad a_{4 i-1,4 i+4}=-s h i 1 \\
& a_{4 i, 4 i-3}=-\operatorname{exi}\left(\beta_{i} c i+\delta_{i} s i\right) \quad a_{4 i, 4 i-2}=-\operatorname{exi}\left(\beta_{i} s i-\delta_{i} c i\right) \\
& a_{4 i, 4 i-1}=-\operatorname{exi}\left(\beta_{i} \operatorname{chi}-\bar{\delta}_{i} \operatorname{sh} i\right) \quad a_{4 i, 4 i}=-\operatorname{exi}\left(\beta_{i} \operatorname{shi}-\bar{\delta}_{i} \operatorname{ch} i\right) \\
& a_{4 i, 4 i+1}=\beta_{i+1} c i 1+\delta_{i+1} s i 1 \quad a_{4 i, 4 i+2}=\beta_{i+1} s i 1-\delta_{i+1} c i 1 \\
& a_{4 i, 4 i+3}=\beta_{i+1} \operatorname{chi} 1+\bar{\delta}_{i+1} \operatorname{shi1} \quad a_{4 i, 4 i+4}=\beta_{i+1} \operatorname{shi1}-\bar{\delta}_{i+1} \operatorname{chi} 1 \\
& a_{4 i+1,4 i-3}=\operatorname{exi}\left[\left(\beta_{i}^{2}-\delta_{i}^{2}\right) c i+2 \beta_{i} \delta_{i} s i\right] \\
& a_{4 i+1,4 i-2}=\operatorname{exi}\left[\left(\beta_{i}^{2}-\delta_{i}^{2}\right) s i-2 \beta_{i} \delta_{i} c i\right] \\
& a_{4 i+1,4 i-1}=\operatorname{exi}\left[\left(\beta_{i}^{2}+\bar{\delta}_{i}^{2}\right) \operatorname{chi}-2 \beta_{i} \bar{\delta}_{i} \operatorname{sh} i\right] \\
& a_{4 i+1,4 i}=\operatorname{exi}\left[\left(\beta_{i}^{2}+\bar{\delta}_{i}^{2}\right) \operatorname{shi}-2 \beta_{i} \bar{\delta}_{i} \operatorname{ch} i\right] \\
& a_{4 i+1,4 i+1}=-2 \beta_{i+1} \delta_{i+1} s i 1+\left(-\beta_{i+1}^{2}+\delta_{i+1}^{2}\right) c i 1 \\
& a_{4 i+1,4 i+2}=2 \beta_{i+1} \delta_{i+1} c i 1+\left(-\beta_{i+1}^{2}+\delta_{i+1}^{2}\right) s i 1 \\
& a_{4 i+1,4 i+3}=2 \beta_{i+1} \bar{\delta}_{i+1} \operatorname{shi1}-\left(\beta_{i+1}^{2}+\bar{\delta}_{i+1}^{2}\right) \operatorname{chi1} \\
& a_{4 i+1,4 i+4}=2 \beta_{i+1} \bar{\delta}_{i+1} \operatorname{chi1}-\left(\beta_{i+1}^{2}+\bar{\delta}_{i+1}^{2}\right) \operatorname{shi1} \\
& a_{4 i+2,4 i-3}=-\operatorname{exi}\left[\left(\beta_{i}^{2}-3 \delta_{i}^{2}\right) \beta_{i} c i+\left(3 \beta_{i}^{2}-\delta_{i}^{2}\right) \delta_{i} s i\right] \\
& a_{4 i+2,4 i-2}=-\operatorname{exi}\left[\left(\beta_{i}^{2}-3 \delta_{i}^{2}\right) \beta_{i} s i+\left(-3 \beta_{i}^{2}+\delta_{i}^{2}\right) \delta_{i} c i\right] \\
& a_{4 i+2,4 i-1}=-\operatorname{exi}\left[\left(\beta_{i}^{2}+3 \bar{\delta}_{i}^{2}\right) \beta_{i} \operatorname{chi}-\left(3 \beta_{i}^{2}+\bar{\delta}_{i}^{2}\right) \bar{\delta}_{i} \operatorname{sh} i\right] \\
& a_{4 i+2,4 i}=-\operatorname{exi}\left[\left(\beta_{i}^{2}+3 \bar{\delta}_{i}^{2}\right) \beta_{i} \operatorname{shi}-\left(3 \beta_{i}^{2}+\bar{\delta}_{i}^{2}\right) \bar{\delta}_{i} \operatorname{chi}\right] \\
& a_{4 i+2,4 i+1}=\left(\beta_{i+1}^{2}-3 \delta_{i+1}^{2}\right) \beta_{i+1} c i 1-\left(-3 \beta_{i+1}^{2}+\delta_{i+1}^{2}\right) \delta_{i+1} s i 1 \\
& a_{4 i+2,4 i+2}=\left(\beta_{i+1}^{2}-3 \delta_{i+1}^{2}\right) \beta_{i+1} s i 1+\left(-3 \beta_{i+1}^{2}+\delta_{i+1}^{2}\right) \delta_{i+1} c i 1 \\
& a_{4 i+2,4 i+3}=-\left(3 \beta_{i+1}^{2}+\bar{\delta}_{i+1}^{2}\right) \bar{\delta}_{i+1} \operatorname{shi} 1+\left(\beta_{i+1}^{2}+3 \bar{\delta}_{i+1}^{2}\right) \beta_{i+1} \operatorname{chi} 1 \\
& a_{4 i+2,4 i+4}=-\left(3 \beta_{i+1}^{2}+\bar{\delta}_{i+1}^{2}\right) \bar{\delta}_{i+1} \operatorname{chi} 1+\left(\beta_{i+1}^{2}+3 \bar{\delta}_{i+1}^{2}\right) \beta_{i+1} \text { shi1 }
\end{aligned}
$$

\section{References}

1. Alshorbagy A.E., Eltaher M.A., Mahmoud F.F., 2011, Free vibration characteristics of a functionally graded beam by finite element method, Applied Mathematical Modelling, 35, 412-425

2. Anandrao K.S., Gupta R.K., Ramachandran P., Rao G.V., 2012, Free vibration analysis of functionally graded beams, Defence Science Journal, 62, 3, 139-146

3. Chauhan P.K., Khan I.A., 2014, Review on analysis of functionally graded material beam type structure, International Journal of Advanced Mechanical Engineering, 4, 3, 299-306

4. Hein H., FekListova L., 2011, Free vibrations of non-uniform and axially functionally graded beams using Haar wavelets, Engineering Structures, 33, 3696-3701

5. HuAng Y., Li X.-F., 2010, A new approach for free vibration of axially functionally graded beams with non-uniform cross-section, Journal of Sound and Vibration, 329, 2291-2303

6. Huang Y., Yang L.-E., Luo Q.-Z., 2013, Free vibration of axially functionally graded Timoshenko beams with non-uniform cross-section, Composites: Part B, 45, 1493-1498

7. Kukla S., Rychlewska J., 2014, Free vibration of axially functionally graded Euler-Bernoulli beams, Journal of Applied Mathematics and Computational Mechanics, 13, 1, 39-44

8. Lebed O.I., Karnovsky I.A., 2000, Formulas for Structural Dynamics, Mc Graw-Hill 
9. Li X.-F., KAng Y.-A., Wu J.-X., 2013, Exact frequency equations of free vibration of exponentially functionally graded beams, Applied Acoustics, 74, 413-420

10. Liu Y., Shu D.W., 2014, Free vibrations analysis of exponential functionally graded beams with a single delamination, Composites: Part B, 59, 166-172

11. Matbuly M.S., Ragb O., Nassar M., 2009, Natural frequencies of a functionally graded cracked beam using the differential quadrature method, Applied Mathematics and Computation, 215, $2307-2316$

12. Pradhan K.K., Chakraverty S., 2013, Free vibration of Euler and Timoshenko functionally graded beams by Rayleigh-Ritz method, Composites: Part B, 51, 175-184

13. Shahba A., Attarnejad R., Zarrinzadeh H., 2013, Free vibration analysis of centrifugally stiffened tapered functionally graded beams, Mechanics of Advanced Materials and Structures, 20, 331-338

14. Shahba A., Rajasekaran S., 2012, Free vibration and stability of tapered Euler-Bernoulli beams made of axially functionally graded materials, Applied Mathematical Modelling, 36, 3094-3111

15. Sina S.A., Navazi H.M., Haddadpour H., 2009, An analytical method for free vibration analysis of functionally graded beams, Materials and Design, 30, 741-747

16. TAng A.-Y., Wu J.-X., Li X.-F., LeE K.Y., 2014, Exact frequency equations of free vibration of exponentially non-uniform functionally graded Timoshenko beams, International Journal of Mechanical Sciences, 89, 1-11

17. Wattanasakulpong N., Ungbhakorn V., 2012, Free vibration analysis of functionally graded beams with general elastically end constraints by DTM, World Journal of Mechanics, 2, 297-310

18. Wu L., Wang Q., Elishakoff I., 2005, Semi-inverse method for axially functionally graded beams with an anti-symmetric vibration mode, Journal of Sound and Vibration, 284, 1190-1202

Manuscript received July 8, 2015; accepted for print December 4, 2015 